Abstract 135 Table 1 Baseline and Follow-up CMR data

\begin{tabular}{lll}
\hline & Baseline & Follow-up \\
\hline LV Septal T1 (ms) & $952(80)$ & $1040(1070)^{* *}$ \\
LV Basal septal T1 (ms) & $953(63)$ & $1042(76)^{* *}$ \\
LV Mid septal TI (ms) & $949(68)$ & $1033(58)^{* *}$ \\
Septal ECV & $0.26(0.06)$ & $0.32(0.04)^{*}$ \\
LV EDVi $\left(\mathrm{ml} / \mathrm{m}^{2}\right)$ & $57(10)$ & $56(10)$ \\
LVESV $\left(\mathrm{ml} / \mathrm{m}^{2}\right)$ & $20(7)$ & $20(8)$ \\
LVEF $(\%)$ & $65(8)$ & $67(5)$ \\
LV Mass index & $54(9)$ & $62(12)$ \\
RVEDV $\left(\mathrm{ml} / \mathrm{m}^{2}\right)$ & $57(10)$ & $56(12)$ \\
RVESV $\left(\mathrm{ml} / \mathrm{m}^{2}\right)$ & $23(6)$ & $21(6)$ \\
RVEF $\left(\mathrm{ml} / \mathrm{m}^{2}\right)$ & $61(7)$ & $63(7)$ \\
\hline
\end{tabular}

Mean (SD), ** $p<0.01$

had LGE; two patients had focal at RV insertion points LGE and two patients had mid-wall LGE in the basal infero-lateral segments.

Conclusion ALMS is associated with increases in ECV and progressive change in T1 values over time that reflects progression of diffuse interstitial fibrosis in asymptomatic adults. Cross-sectional studies have identified ECV as a biomarker of cardiovascular "vulnerability" but longitudinal tracking has the potential to highlight those at greatest risk.

\section{BEYOND THE AORTA: EXPERIENCES OF NEUROVASCULAR IMAGING IN LOEYS-DIETZ AND VASCULAR EHLERS DANLOS SYNDROME}

Carolyn Smith*, Sarju Mehta, Nigel Burrows, Peter Martin, Rosemary Rusk. Addenbrookes Hospital; *Presenting Author

\subsection{6/heartjnl-2016-309890.136}

Introduction The vascular complications of inherited syndromes Loeys-Dietz (LDS) and Vascular Ehlers Danlos (vEDS) lead to significant morbidity and mortality. Current recommendations advocate head to pelvis screening for LDS, and to a lesser extent for vEDS as complications typically involve medium to large arteries. We reviewed the practice in our aortopathy Inherited Cardiac Conditions Clinic (AICC). In our institution these patients are managed by a multi-specialist approach including cardiologists, neurologists, vascular surgeons, chest physicians, dermatologists and geneticists.

Methods Patients were identified through current attendance at AICC or discussion at the linked multidisciplinary team (MDT) meeting. Electronic notes and imaging were reviewed to determine clinical details, genetic diagnosis, screening offered and (neuro) vascular complications.

Results 36 patients were identified. Of these: 14 had a genetic diagnosis of vEDS, 8 a genetic diagnosis of LDS, 4 were excluded (alternate genetic diagnosis e.g. Marfan's) and the remaining 10 had a suggestive clinical phenotype without a typical LDS/vEDS gene mutation and were reviewed alongside the LDS/vEDS population. Screening was performed with separate CT angiograms (arch to carotids and arch to iliacs) to obtain adequate image quality.

Two patients had clinical neurovascular events (spontaneous dissection). To date neurovascular imaging has been offered to 27 of the 32 patients $(82 \%)$. Of these, two patients declined screening, leading to $78 \%$ of the patient group being studied (25 of 32 ).
Neurovascular involvement of all patients screened reached $52 \%$ (4 vEDS, 4 LDS, 5 other) including a high proportion of the patients (5 of 6 screened) without genetic LDS/vEDS diagnosis (Tables 1, 2; Figure 1). Significant neurovascular pathology was identified in the absence of aortic pathology, including one LDS patient with normal aortic dimensions and morphology, a finding not commonly reported in the literature.

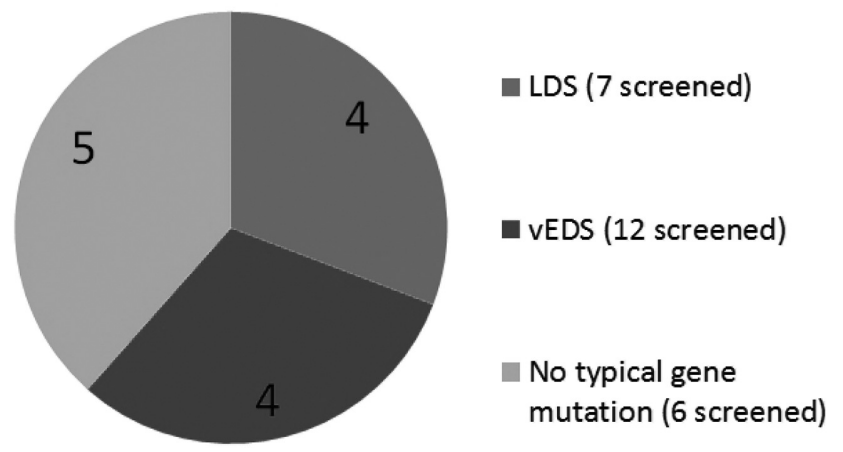

Abstract 136 Figure 1 Neurovascular involvement by diagnosis

Conclusions This is a small population of patients, but the neurovascular involvement rate is high. We would recommend that head to pelvis screening is considered for all LDS and vEDS patients, including those with normal aortic dimensions and morphology. Furthermore, this data implies that patients with a suggestive clinical phenotype should be offered the same screening protocols. It also emphasises the importance of a multi-specialist approach to these patients and their management should not be confined to cardiology alone. Once identified, the specialist MDT is key in making decisions on further surveillance and treatment threshold.

\section{PARADOXICAL EMBOLISM RISK INCREASES WITH ATYPICAL RIGHT ATRIAL BLOOD FLOW IN THE PRESENCE OF A PATENT FORAMEN OVALE}

${ }^{1}$ Victoria Stoll ${ }^{*},{ }^{1}$ Aaron Hess, ${ }^{1}$ Oliver Rider, ${ }^{1}$ Hayley Harvey, ${ }^{1}$ Alex Pitcher, ${ }^{1}$ Margaret Loudon, ${ }^{1}$ Malenka Bissell, 'Stefan Neubauer, ${ }^{2}$ Oliver Ormerod, 'Saul Myerson. 'OCMR, University of Oxford; ${ }^{2}$ Oxford University Foundation Trust; *Presenting Author

\subsection{6/heartjnl-2016-309890.137}

Abstract 136 Table 1 Positive neurovascular imaging results in LDS/vEDS; (ICA, internal carotid artery; TS Trans Sinus; AA Ascending Aorta)

\begin{tabular}{llll}
\hline Diagnosis & Age & Neurovascular & Aorta (cm) \\
\hline LDS & 77 & Left ICA aneurysm multiple vessel ectasia & Dilated TS 4.7 AA 3.8 \\
LDS & 61 & Right ICA aneurysm, tortuosity & Normal TS 3.6 AA 2.4 \\
LDS & 53 & Left middle cerebral artery ectasia & Operated: AA graft \\
LDS & 29 & Right ICA dilatation (?previous dissection) & Operated: AA graft \\
vEDS & 35 & Left ICA pseudoaneurysm & Normal TS 2.4 AA 2.4 \\
vEDS & 38 & Right ICA dilatation (?previous dissection) & Normal TS 2.8 AA 2.3 \\
vEDS & 31 & Bilateral ICA aneurysm and dissection & Dilated TS 3.9 AA 3.1 \\
vEDS & 43 & Left vertebral artery pseudoaneurysm & Normal AA 3.3 \\
\hline
\end{tabular}

